OKHEP-01-03R

hep-ph/0105250

July 2001

\title{
Constraints on extra dimensions from cosmological and terrestrial measurements
}

\author{
Kimball A. Milton, ${ }^{*}$ Ronald Kantowski, ${ }^{\dagger}$ Chung Kao, ${ }^{\ddagger}$ and Yun Wang ${ }^{\S}$ \\ Department of Physics and Astronomy, The University of Oklahoma, Norman, OK 73019
}

(October 30, 2018)

\begin{abstract}
If quantum fields exist in extra compact dimensions, they will give rise to a quantum vacuum or Casimir energy. That vacuum energy will manifest itself as a cosmological constant. The fact that supernova and cosmic microwave background data indicate that the cosmological constant is of the same order as the critical mass density to close the universe supplies a lower bound on the size of the extra dimensions. Recent laboratory constraints on deviations from Newton's law place an upper limit. The allowed region is so small as to suggest that either extra compact dimensions do not exist, or their properties are about to be tightly constrained by experimental data.
\end{abstract}

*Email: milton@physics.ou.edu

$\dagger$ Email: ski@physics.ou.edu

‡Email: kao@physics.ou.edu

§Email: wang@physics.ou.edu 
It has been appreciated for many years that there is an apparently fundamental conflict between quantum field theory and the smallness of the cosmological constant [1]. This is because the zero-point energy of the quantum fields (including gravity) in the universe should give rise to an observable cosmological vacuum energy density,

$$
u_{\mathrm{cosmo}} \sim \frac{1}{L_{\mathrm{Pl}}^{4}}
$$

where the Planck length is

$$
L_{\mathrm{Pl}}=\sqrt{G_{N}}=1.6 \times 10^{-33} \mathrm{~cm} .
$$

(We use natural units with $\hbar=c=1$. The conversion factor is $\hbar c \simeq 2 \times 10^{-14} \mathrm{GeV} \mathrm{cm}$.) This means that the cosmic vacuum energy density would be

$$
u_{\text {cosmo }} \sim 10^{118} \mathrm{GeV} \mathrm{cm}^{-3},
$$

which is 123 orders of magnitude larger than the critical mass density required to close the universe:

$$
\rho_{c}=\frac{3 H_{0}^{2}}{8 \pi G_{N}}=1.05 \times 10^{-5} h_{0}^{2} \mathrm{GeV} \mathrm{cm}^{-3},
$$

in terms of the dimensionless Hubble constant, $h_{0}=H_{0} / 100 \mathrm{~km} \mathrm{~s}^{-1} \mathrm{Mpc}^{-1}$. From relativistic covariance the cosmological vacuum energy density must be the 00 component of the expectation value of the energy-momentum tensor, which we can identify with the cosmological constant:

$$
\left\langle T^{\mu \nu}\right\rangle=-u g^{\mu \nu}=-\frac{\Lambda}{8 \pi G} g^{\mu \nu} .
$$

[We use the metric with signature $(-1,1,1,1)$.] Of course this is absurd with $u$ given by Eq. (3), which would have caused the universe to expand to zero density long ago.

For most of the past century, it was the prejudice of theoreticians that the cosmological constant was exactly zero, although no one could give a convincing argument. Recently, however, with the new data gathered on the brightness-redshift relation for very distant type Ia supernovæ [2,3], corroborated by the balloon observations of the anisotropy in the cosmic microwave background [4 6], it seems clear that the cosmological constant is near the critical value, or $\Omega_{\Lambda}=\Lambda / 8 \pi G \rho_{c} \simeq 0.6-0.7$ [ [4, 6]. It is very hard to understand how the cosmological constant can be nonzero but small.

We here present a plausible scenario for understanding this puzzle. It is reasonable (but by no means established) that vacuum fluctuations in the gravitational and matter fields in flat Minkowski space give a zero cosmological constant. (See below.) Effects due to

\footnotetext{
${ }^{1}$ This is in line with considerations of Casimir energies in other contexts. For example, although the electromagnetic Casimir energy of a ball of dilute nondispersive dielectric material is divergent, that divergence can be unambiguously removed as an unobservable bulk and surface effect, and a unique finite energy, interpretable as a sum of van der Waals energies, emerges. See Refs. [7].
} 
curvature are negligible. But since the work of Kaluza and Klein [8] it has been an exciting possibility that there exist extra dimensions beyond those of Minkowski space-time. Why do we not experience those dimensions? The simplest possibility seems to be that those extra dimensions are curled up in a space $\mathcal{S}$ of size $a$, smaller than some observable limit.

Of course, in recent years, the idea of extra dimensions has become much more compelling. Superstring theory requires at least 10 dimensions, six of which must be compactified, and the putative $M$ theory, supergravity, is an 11 dimensional theory. Perhaps, if only gravity experiences the extra dimensions, they could be of macroscopic size. Various scenarios have been suggested [9, 10].

Macroscopic extra dimensions imply deviations from Newton's law at such a scale. A year ago, millimeter scale deviations seemed plausible, and many theorists hoped that the higher-dimensional world was on the brink of discovery. Experiments were initiated [11]. Very recently, the results of the first definitive experiment have appeared [12], which indicate no deviation from Newton's law down to $218 \mu \mathrm{m}$. This poses a serious constraint for modelbuilders.2

Here we propose that a very tight constraint indeed emerges if we recognize that compact dimensions of size a necessarily possess a quantum vacuum or Casimir energy of order $u(z) \sim a^{-4}$. That such energies are observable is confirmed by recent experiments [14]. These can be calculated in simple cases. Applequist and Chodos [15] found that the Casimir energy for the case of scalar field on a circle, $\mathcal{S}=S^{1}$, was

$$
u_{C}=-\frac{3 \zeta(5)}{64 \pi^{6} a^{4}}=-\frac{5.056 \times 10^{-5}}{a^{4}},
$$

which needs only to be multiplied by 5 for graviton fluctuations. The general case of scalars on $\mathcal{S}=S^{N}, N$ odd, was considered by Candelas and Weinberg [16], who found that the Casimir energy was positive for $3 \leq N \leq 19$, with a maximum at $N=13$ of $u_{C}=1.374 \times 10^{-3} / a^{4}$. The even dimensional case was much more subtle, because it was divergent. Kantowski and Milton [17] showed that the coefficient of the logarithmic divergence was unique, and adopting the Planck length as the natural cutoff, found

$$
S^{N}, N \text { even : } \quad u_{C}^{N}=\frac{\alpha_{N}}{a^{4}} \ln \frac{a}{L_{\mathrm{Pl}}},
$$

but $\alpha_{N}$ was always negative for scalars. In a second paper [18] they extended the analysis to vectors, tensors, fermions, and to massive particles, among which cases positive values of the (divergent) Casimir energy could be found. Some representative results for massless

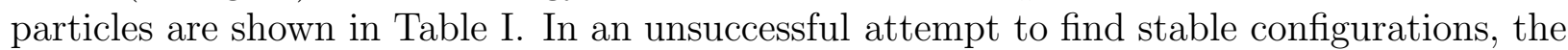
analysis was extended to cases where the internal space was the product of spheres [21].

It is important to recognize that these Casimir energies correspond to a cosmological constant in our $3+1$ dimensional world, not in the extra compactified dimensions or "bulk." They constitute an effective source term in the 4-dimensional Einstein equations. That

\footnotetext{
${ }^{2}$ We might also mention short distance constraints on Yukawa-type corrections to the gravitational potential coming from Casimir measurements themselves [13].
} 
there is a correlation between the currently favored value of the cosmological constant and submillimeter-sized extra dimensions has been noted qualitatively before 22,23].

The goal, of course, in all these investigations was to include graviton fluctuations. However, it immediately became apparent that the results were gauge- and reparameterizationdependent unless the DeWitt-Vilkovisky formalism was adopted [24]. This was an extraordinarily difficult task. Only in the past year has the general analysis for gravity appeared, with results for a few special geometries [26]. Cho and Kantowski obtain the unique divergent part of the effective action for $\mathcal{S}=S^{2}, S^{4}$, and $S^{6}$, as polynomials in $\Lambda a^{2}$. (Unfortunately, once again, they are unable to find any stable configurations.) The results are also shown in Table [1, for $\Lambda a^{2} \sim G / a^{2} \ll 1$. It will be noted that graviton fluctuations dominate matter fluctuations, except in the case of a large number of matter fields in a small number of dimensions. Of course, it would be very interesting to know the graviton fluctuation results for odd-dimensional spaces, but that seems to be a more difficult calculation; it is far easier to compute the divergent part than the finite part, which is all there is in odd-dimensional spaces.

These generic results may be applied to recent popular scenarios. For example, in the ADD scheme [9] only gravity propagates in the bulk, while the RS approach [10] has other bulk fields in a single extra dimension.

Let us now perform some simple estimates of the cosmological constant in these models. The data suggest a positive cosmological constant, so we can exclude those cases where the Casimir energy is negative. For the odd $N$ cases, where the Casimir energy is finite, let us write

$$
S^{N}, \quad N \text { odd }: \quad u_{C}^{N}=\frac{\beta_{N}}{a^{4}}
$$

so merely requiring that this be less than the critical density $\rho_{c}$ implies $(\beta>0)$

$$
a \gtrsim \beta^{1 / 4} h_{0}^{-1 / 2} 67 \mu \mathrm{m} \approx \beta^{1 / 4} 80 \mu \mathrm{m},
$$

taking [27] $h_{0}=0.7$ (with about a 10-20\% uncertainty). As seen in Table II these lower limits (for a single species) are still an order of magnitude below the experimental upper limit [12]. Much tighter constraints appear if we use the divergent results for even dimensions. We have the inequality $(\alpha>0)$

$$
a \gtrsim\left[\alpha \ln \left(a / L_{\mathrm{Pl}}\right)\right]^{1 / 4} 80 \mu \mathrm{m}
$$

where we can approximate $\left(\ln a / L_{\mathrm{Pl}}\right)^{1 / 4} \approx 2.9$. Again results are shown in Table III, which rules out all but one of the gravity cases $\left(S^{2}\right)$ given by Cho and Kantowski [26]. For matter fluctuations only [18, excluded are $N>14$ for a single vector field and $N>6$ for a single tensor field. (Fermions always have a negative Casimir energy in even dimensions.) Of course, it is possible to achieve cancellations by including various matter fields and

\footnotetext{
${ }^{3}$ A few special cases were known earlier. Besides that of $\mathcal{S}=S^{1}$, the general six-dimensional background was considered by Cho and Kantowski [25], which includes $\mathcal{S}=S^{1} \times S^{1}$ and $S^{2}$.
} 
gravity. In general the Casimir energy is obtained by summing over the species of field which propagate in the extra dimensions,

$$
u_{\mathrm{tot}}=\frac{1}{a^{4}} \sum_{i}\left[\alpha_{i} \ln \left(a / L_{\mathrm{Pl}}\right)+\beta_{i}\right] \approx \frac{\beta_{\mathrm{eff}}}{a^{4}},
$$

which leads to a lower limit according to Eq. (9). Presumably, if exact supersymmetry held in the extra dimensions (including supersymmetric boundary conditions), the Casimir energy would vanish, but this would seem to be difficult to achieve with large extra dimensions (1 mm corresponds to $2 \times 10^{-4} \mathrm{eV}$.)

It seems to be commonly believed that submillimeter tests of gravity put no limits on the size of extra dimensions if $N>2$. This is because of the relation of the size $R$ of the extra dimensions in the ADD scheme to the fundamental $4+N$ gravity scale $M$ [28]:

$$
R \sim \frac{1}{M}\left(\frac{M_{\mathrm{Pl}}}{M}\right)^{2 / N},
$$

where $M_{\mathrm{Pl}}=1 / L_{\mathrm{Pl}}=1.2 \times 10^{16} \mathrm{TeV}$ is the usual Planck mass. Moreover, the supernova limits on ADD extra dimensions (due to production of Kaluza-Klein gravitons) become rapidly smaller with increase in $N$ [29]:

$$
\begin{array}{ll}
N=2: & R<0.9 \times 10^{-4} \mathrm{~mm}, \\
N=3: & R<1.9 \times 10^{-7} \mathrm{~mm} .
\end{array}
$$

Thus direct tests of Newton's law are not competitive. However, the resulting Casimir contribution to the cosmological constant would be enormous for such small compactified regions, and it would seem impossible to naturally resolve this problem.

The situation at first glance seems rather different with the RS scenario. In the original scheme, gravity is localized in the "Planck brane," while the standard-model particles are confined to the "TeV brane." As a consequence, it might appear that the quantum fluctuations of both brane and bulk fields are negligible [30]. It has been stated that the cosmological constant becomes exponentially small as the brane separation becomes large [31. However, this is at the "classical level," without bulk fluctuations; explicit considerations show that quantum effects give rise to a large cosmological constant, of order of that given by Eq. (3), unless an appeal is made to fine tuning [32]. Moreover, if the scenario is extended so that the world brane contains compactified dimensions in which gravity lives [33, the constraints we deduce here directly apply.

There have been a number of proposals in which either in string [34, 35] or brane [36] contexts the cosmological constant can be made to vanish. These solutions may not be altogether natural, and may indeed require fine tuning [37]. (Moreover, the suggestions either do not include gravitational fluctuations, or ignore the problem of gauge and parameterization dependence.) Such ideas could explain the vanishing of $u_{\text {cosmo, }}$, or of a corresponding energy arising at the electroweak symmetry breaking scale, $\Lambda_{\mathrm{EW}} \sim 1 \mathrm{TeV}$,

$$
u_{\mathrm{EW}} \sim \Lambda_{\mathrm{EW}}^{4} \sim 10^{53} \mathrm{GeV} / \mathrm{cm}^{3},
$$

due to fluctuations in standard model fields, but they would presumably not lead to the simultaneous vanishing of the Casimir energy. Indeed Sundrum in Ref. 34 obtains a small 
cosmological constant based on a narrow window in allowed graviton compositeness, or string, scale, $m_{\mathrm{st}}$,

$$
10 \mu \mathrm{m}<\frac{1}{m_{\mathrm{st}}}<1 \mathrm{~cm}
$$

Theoretical and experimental limits this past year have nearly closed this window. Clearly, the ideas expressed here provide a stringent constraint for model builders.

\section{ACKNOWLEDGMENTS}

This research was supported in part by the U.S. Department of Energy under Grant No. DE-FG03-98ER41066 and by the National Science Foundation CAREER grant AST0094335. 


\section{REFERENCES}

[1] S. Weinberg, Rev. Mod. Phys. 61, 1 (1989); Phys. Rev. D 61, 103505 (2000); talk at Dark Matter 2000, Marina del Rey, CA, 2000, astro-ph/0005265.

[2] P.M. Garnavich et al., Astrophys. J. Lett. 493, L53 (1998); A. G. Reiss et al., Astron. J. 116, 1009 (1998).

[3] S. Perlmutter et al., Astrophys. J. 517, 565 (1999).

[4] The BOOMERANG collaboration: P. de Bernardis et al., Nature, 404, 955 (2000); A. E. Lange et al., Phys. Rev. D 63, 042001 (2001); C. B. Netterfield et al., astro$\mathrm{ph} / 0104460$.

[5] The MAXIMA collaboration: A. Balbi et al., Astrophys. J. Lett. 545, L1 (2000); S. Hanany et al., Astrophys. J. Lett. 545, L5 (2000).

[6] The DASI collaboration: C. Pryke et al., astro-ph/0104490.

[7] K. A. Milton and Y. J. Ng, Phys. Rev. E 57, 5504 (1998); I. Brevik, V. N. Marachevsky, and K. A. Milton, Phys. Rev. Lett. 82, 3948 (1999); G. Barton, J. Phys. A 32, 525 (1999); M. Bordag, K. Kirsten, and D. Vassilevich, Phys. Rev. D 59, 085011 (1999); J. S. Høye and I. Brevik, J. Stat. Phys. 100, 223 (2000).

[8] T. Kaluza, Sitz. Preuss. Akad. Wiss. Phys. Math. K1, 966 (1921); O. Klein, Nature 118, 516 (1926); Z. Phys. 37, 895 (1926).

[9] N. Arkani-Hamed, S. Dimopoulos and G. Dvali, Phys. Lett. B 429, 263 (1998); I. Antoniadis, N. Arkani-Hamed, S. Dimopoulos and G. Dvali, Phys. Lett. B 436, 257 (1998).

[10] L. Randall and R. Sundrum, Phys. Rev. Lett. 83, 3370 (1999); Phys. Rev. Lett. 83, 4690 (1999).

[11] J. C. Long, H. W. Chan, and J. C. Price, Nucl. Phys. B 539, 23 (1999); J. C. Long, A. B. Churnside, and J. C. Price, in Proceedings of the 9th Marcel Grossmann Meeting on Recent Developments in Theoretical and Experimental General Relativity, Gravitation, and Relativistic Field Theories (MG9), Rome, 2-9 July 2000, hep-ph/0009062.

[12] C. D. Hoyle, U. Schmidt, B. R. Heckel, E. G. Adelberger, J. H. Gundlach, D. J. Kapner, and H. E. Swanson, Phys. Rev. Lett. 86, 1418 (2001).

[13] M. Bordag, B. Geyer, G. L. Klimchitskaya, and V. M. Mostepanenko, Phys. Rev. D 58, 075003 (1998); 60, 055004 (1999); 62, 011701(R) (2000); V. M. Mostepaneko and M. Novello, ibid. 63, 115003 (2001); E. Fischbach, D.E. Krause, V.M. Mostepanenko, M. Novello, hep-ph/0106331.

[14] S. K. Lamoreaux, Phys Rev. Lett. 78, 5 (1997); 81, 5475(E) (1998); U. Mohideen and A. Roy, Phys. Rev. Lett. 81, 4549 (1998); 83, 3341 (1999); A. Roy, C.-Y. Lin, and U. Mohideen, Phys. Rev. D 60, R111101 (1999); B. W. Harris, F. Chen, and U. Mohideen, Phys. Rev. A 62, 052109 (2000); H. B. Chan, V. A. Aksyuk, R. N. Kleiman, D. J. Bishop, and F. Capasso, Science 291, 1941 (2001) (10.1126/science.1057984).

[15] T. Appelquist and A. Chodos, Phys. Rev. Lett. 50, 141 (1983); Phys. Rev. D 28, 772 (1983).

[16] P Candelas and S. Weinberg, Nucl. Phys. B 237, 397 (1984).

[17] R. Kantowski and K. A. Milton, Phys. Rev. D 35, 549 (1987).

[18] R. Kantowski and K. A. Milton, Phys. Rev. D 36, 3712 (1987).

[19] A. Chodos and E. Myers, Phys. Rev. D 31, 3064 (1985).

[20] M. H. Sarmadi, Nucl. Phys. B 263, 187 (1986).

[21] D. Birmingham, R. Kantowski, and K. A. Milton, Phys. Rev. D 38, 1809 (1988). 
[22] D. B. Kaplan and M. B. Wise, JHEP 08, 037 (2000); T. Banks, Nucl. Phys. B 309, 493 (1988); S. R. Beane, Phys. Lett. B 358, 203 (1995); Gen. Rel. Grav. 29, 945 (1997).

[23] J.-W. Chen, M. A. Luty, and E. Pontón, JHEP 09, 012 (2000).

[24] G. A. Vilkovisky, in Quantum Theory of Gravity, ed. S. C. Christensen (Hilger, Bristol, 1984); Nucl. Phys. B 234, 125 (1984); A. O. Barvinsky and G. A. Vilkovisky, Phys. Rep. 119, 1 (1985); B. S. DeWitt, in Quantum Field Theory and Quantum Statistics, ed. I. A. Batalin, C. J. Isham, and G. A. Vilkovisky (Hilger, Bristol, 1987).

[25] H. T. Cho and R. Kantowski, Phys. Rev. Lett. 67, 422 (1991); 75, 2630(E) (1995).

[26] H. T. Cho and R. Kantowski, Phys. Rev. D 62, 124003 (2000).

[27] Particle Data Group, Eur. Phys. J. C 15, 1 (2000); W. J. Freedman et al., astroph/0012376, Ap. J., to be published; D. Branch, Annu. Rev. Astron. Astrophys. 36, 17 (1998), astro-ph/9801065; and references therein.

[28] N. Arkani-Hamed, S. Dimopoulos, and G. Divali, Phys. Rev. D 59, 086004 (1999); G. F. Guidice, R. Rattazzi, and J. D. Wells, Nucl. Phys. B 5443 (1999); E. A. Mirabelli, M. Perelstein, and M. E. Peshkin, Phys. Rev. Lett. 82, 2236 (1999).

[29] S. Hannestad and G. Raffelt, hep-ph/0103201; V. Barger, T. Han, C. Kao and R. J. Zhang, Phys. Lett. B 461, 34 (1999); S. Cullen and M. Perelstein, Phys. Rev. Lett. 83, 268 (1999).

[30] W. D. Goldberger and I. Z. Rothstein, Phys. Lett. B 475, 275 (2000); I. Brevik, K. A. Milton, S. Nojiri, and S. D. Odintsov, Nucl. Phys. B 599, 305 (2001).

[31] S.-H. H. Tye and I. Wasserman, Phys. Rev. Lett. 86, 1682 (2001).

[32] S. Nojiri, O. Obregon, and S. D. Odintsov, hep-th/0105300.

[33] Z. Chako, P. J. Fox, A. E. Nelson, and N. Weiner, hep-ph/0106343.

[34] R. Sundrum, JHEP 9907, 001 (1999).

[35] S. Kachru and E. Silverstein, JHEP 01, 004 (1999); S. Kachru, J. Kumar, and E. Silverstein, Phys. Rev. D 59, 106004 (1999); S. Kachru, M. Schulz, and E. Silverstein, Phys. Rev. D 62, 045021 (2000).

[36] N. Arkani-Hamed, S. Dimopoulos, N. Kaloper, and R. Sundrum, Phys. Lett. B 480, 193 (2000); S. Förste, Z. Lalak, S. Lavignac, and H. P. Nilles, Phys. Lett. B 481, 360 (2000).

[37] S. Förste, Z. Lalak, S. Lavignac, and H. P. Nilles, JHEP 09, 034 (2000). 


\section{TABLES}

\begin{tabular}{lcccc}
\hline \hline Geometry $\mathcal{S}$ & Gravity & Scalar & Fermion & Vector \\
\hline$S^{1}(\mathrm{u})$ & $\beta=-2.53 \times 10^{-4}$ & $-5.06 \times 10^{-5}$ & $2.02 \times 10^{-4}$ & - \\
$S^{1}(\mathrm{t})$ & $\beta=2.37 \times 10^{-4}$ & $4.74 \times 10^{-5}$ & $-1.90 \times 10^{-4}$ & - \\
$S^{2}$ & $\alpha=1.70 \times 10^{-2}$ & $-8.04 \times 10^{-5}$ & $-7.94 \times 10^{-4}$ & $-8.04 \times 10^{-5}$ \\
$S^{3}$ & - & $\beta=7.57 \times 10^{-5}$ & $1.95 \times 10^{-4}$ & - \\
$S^{4}$ & $\alpha=-0.489$ & $-4.99 \times 10^{-4}$ & $-6.64 \times 10^{-3}$ & $1.21 \times 10^{-2}$ \\
$S^{5}$ & - & $\beta=4.28 \times 10^{-4}$ & $-1.14 \times 10^{-4}$ & - \\
$S^{6}$ & $\alpha=5.10$ & $-1.31 \times 10^{-3}$ & $-3.02 \times 10^{-2}$ & $4.90 \times 10^{-2}$ \\
$S^{7}$ & - & $\beta=8.16 \times 10^{-4}$ & $5.96 \times 10^{-5}$ & - \\
\hline \hline
\end{tabular}

TABLE I. The Casimir energy for $M^{4} \times \mathcal{S}$ is tabulated for various field types in the compact geometry $\mathcal{S}$. We write $u=\left[\alpha \ln \left(a / L_{\mathrm{Pl}}\right)+\beta\right] a^{-4}$, and give $\alpha$ for even internal dimension and $\beta$ for odd, where $\alpha=0$. For $S^{1} \mathrm{u}$ denotes untwisted (periodic) while t twisted (antiperiodic) boundary conditions. The entries marked with dashes have not been calculated. (We should note that a general recipe for calculating the odd- $N$ terms for vectors and tensors is given in Ref. [19], but results are not explicit, and require knowledge of the "polylogarithmic-exponential" function. Moreover, the Casimir energies they find are complex. The method given in Ref. [18] has, in contrast, no problems with tachyons, and would give real energies. Explicit numbers given in Ref. 20] for the various components of gravity without the necessary Vilkovisky-DeWitt correction do not allow one to extract the transverse vector part without further calculation.) The numbers are taken from Refs. 16 18,26].

\begin{tabular}{lcccc}
\hline \hline Geometry $\mathcal{S}$ & Gravity & Scalar & Fermion & Vector \\
\hline$S^{1}(\mathrm{u})$ & $*$ & $*$ & $9.5 \mu \mathrm{m}$ & - \\
$S^{1}(\mathrm{t})$ & $9.9 \mu \mathrm{m}$ & $6.6 \mu \mathrm{m}$ & $*$ & - \\
$S^{2}$ & $84 \mu \mathrm{m}$ & $*$ & $*$ & $*$ \\
$S^{3}$ & - & $7.5 \mu \mathrm{m}$ & $9.5 \mu \mathrm{m}$ & - \\
$S^{4}$ & $*$ & $*$ & $*$ & $77 \mu \mathrm{m}$ \\
$S^{5}$ & - & $11.5 \mu \mathrm{m}$ & $*$ & - \\
$S^{6}$ & $350 \mu \mathrm{m}$ & $*$ & $*$ & $110 \mu \mathrm{m}$ \\
$S^{7}$ & - & $13.5 \mu \mathrm{m}$ & $7.0 \mu \mathrm{m}$ & - \\
\hline \hline
\end{tabular}

TABLE II. The lower limit to the radius of the compact dimensions deduced from the requirement that the Casimir energy not exceed the critical density. The numbers shown are for a single species of the field type indicated. The dashes indicate cases where the Casimir energy has not been calculated, while asterisks indicate (phenomenologically excluded) cases where the Casimir energy is negative. 